4

\title{
Smartphone-interrogated test supports for the enzymatic determination of putrescine and cadaverine in food
}

\author{
Isabel Sanz-Vicente ${ }^{1}$. Ángel López-Molinero ${ }^{2}$ - Susana de Marcos ${ }^{1}$ • Jesús Navarro ${ }^{2}$. Pablo Cebrián ${ }^{3}$. \\ Chantal Arruego ${ }^{3} \cdot$ Victor Visiedo $^{3}$. Javier Galbán ${ }^{1}$
}

Received: 21 February 2020 /Revised: 8 April 2020 / Accepted: 21 April 2020

(C) Springer-Verlag GmbH Germany, part of Springer Nature 2020

\begin{abstract}
Diamino-oxidase (DAO), horseradish peroxidase (HRP), and tetramethylbenzidine (TMB) have been immobilized into cellulose to obtain circular cellulose test supports (CCTSs) for the determination of cadaverine (Cad) and putrescine (Put). During the enzymatic reaction, TMB is oxidized and a blue spot is obtained. This color (RGB coordinates) is measured with a smartphone and a commercial application. The highest sensitivity is provided by the component $R$ and a linear response is observed for low biogenic amine (BA) concentrations, but a second-order polynomial response better fits the experimental results for a wider concentration range. This has been successfully explained with a model developed to explain the RGB values obtained in this type of analytical system. Optimization studies enable CCTSs to be obtained for Put and Cad determination, which could be used (kept at $4{ }^{\circ} \mathrm{C}$ ) for at least 45 days if a stabilizer (StabilCoat ${ }^{\mathrm{TM}}$ or StabilGuard ${ }^{\mathrm{TM}}$ ) is added during its synthesis. In these conditions, the $R$ coordinate follows the model up to at least $4 \times 10^{-4} \mathrm{M}$ Put and/or Cad (both analytes give the same response). The method permits the Put and Cad determination from $5 \times 10^{-5} \mathrm{M}$ up to $4 \times 10^{-4} \mathrm{M}(\mathrm{RSD}=3 \%, n=3)$. The CCTSs have been applied to $\mathrm{Put}+\mathrm{Cad}$ determination in a tuna sample without any interference by other biogenic amines. The concentration found statistically agrees with that obtained using a HPLC-MS-validated method.
\end{abstract}

Keywords Putrescine $\cdot$ Cadaverine $\cdot$ Diamine oxidase $\cdot$ TMB $\cdot$ Test supports $\cdot$ Cellulose

Parts of this work were presented at the X Iberian Spectroscopy Conference (X CIE) and the XXVI National Spectroscopy Meeting (RNE) (Spain) in July 2018 and have been awarded with an ABC Best Poster Award.

Electronic supplementary material The online version of this article (https://doi.org/10.1007/s00216-020-02677-7) contains supplementary material, which is available to authorized users.

Isabel Sanz-Vicente

isasanz@unizar.es

1 Nanosensors and Bioanalytical Systems (N\&SB), Analytical Chemistry Department, Faculty of Sciences, Aragon Institute of Nanoscience, University of Zaragoza, 50009 Zaragoza, Spain

2 Nanosensors and Bioanalytical Systems (N\&SB), Analytical Chemistry Department, Faculty of Sciences, University of Zaragoza, 50009 Zaragoza, Spain

3 Analytical Chemistry Department, Faculty of Sciences, University of Zaragoza, 50009 Zaragoza, Spain
Introduction

Biogenic amines (BAs) are organic molecules having important biological activity, especially as neurotransmitters or hormones. The human body can enzymatically synthesize them, usually through decarboxylation of the corresponding amino acids (amino acid decarboxylases, AADCs). They are also present in many types of foodstuffs, where they appear by bacterial AADC activity; their concentrations are especially high in foods with a high protein content (e.g., meat, fish, or dairy products) or in those whose storage (temperature, $\mathrm{pH}$, salinity, packaging) or processing (fermentation) conditions allow the bacteria involved to proliferate. Consequently, the concentration of BAs is an indication of freshness and quality of both food and raw materials. The human body has a mechanism for elimination of BAs by means of enzymatic reactions catalyzed by diamino-oxidase (DAO), monoamino-oxidase (MAO), and histamine $N$-methyltransferase (HNMT). Nevertheless, in certain individuals, these reactions may be insufficient due to low enzymatic activity or the use of drugs 
that inhibit those enzymes. This can cause several health disorders ranging from intolerance to intoxication [1].

From a toxicological point of view, the most studied BAs are histamine (His) (the only one for which there is legislation) and tyramine (Tyr). However, there is a growing interest in the study of some polyamines, especially cadaverine (Cad) and putrescine (Put). First, they are also natural substrates of DAO and consequently reduce the ability of this enzyme to degrade His and Tyr; second, they are responsible for the formation of nitrosamides (recognized carcinogens) when combined with nitrites, for instance during cooking [2]; and third, they are indicators of tumoral processes [3-5].

The usual methods to determine these compounds are based on separation techniques such as gas chromatography, thin-layer chromatography, or high-performance liquid chromatography [6-8]. They give good results, although they need time-consuming sample preparation and derivatization stages. Food sector industries and public health agencies are interested in having, at their disposal, faster and versatile quality control systems, so they need easy, cheap, and fast analytical methods directed towards specific BAs.

An alternative method consists of using enzymatic methods in solution. This is based on the scheme developed by Lerke et al. [9] for histamine determination (Scheme 1). In the first step, an amino-oxidase enzyme (in our case, DAO) catalyzes the loss of an amino group of the BAs (in this study, $\mathrm{Cad}$ or Put) forming the corresponding aldehyde and $\mathrm{H}_{2} \mathrm{O}_{2}$. In the second step, the horseradish peroxidase (HRP) enzyme catalyzes the oxidation of a chemical transducer by $\mathrm{H}_{2} \mathrm{O}_{2}$. This scheme has been successfully used for developing electrochemical sensors using different types of charge transfer mediators [10-13].

The same scheme has been used for the development of a solution colorimetric method for BAs, replacing the charge transfer mediator by a chromogen, such as $2,2^{\prime}$-azino-bis(3ethylbenzothiazoline-6-sulfonic acid) diammonium salt (ABTS) $[14,15]$. However, more interesting is the development of test strips after the immobilization of enzymes on paper or cellulose [16]. Following this idea, Hall et al. [17]

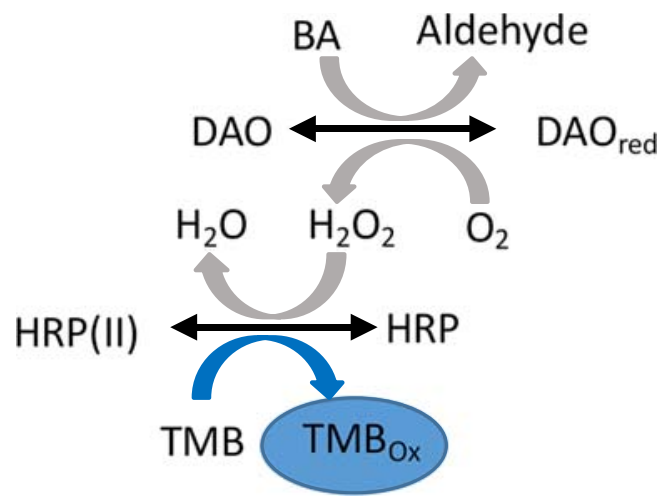

Scheme 1 Sequence on the enzymatic reactions used for BPA determination dipped commercial peroxide test strips in a mixture of DAO and HRP, making them sensitive to histamine, and later to Cad and Put [18]. These strips have not been commercialized, perhaps because the measurement time is between 7 and 10 min. In addition, the increase in absorbance that occurs at $560 \mathrm{~nm}$ was measured by reflectance with a spectrophotometer, which hindered the possibility of quantitative measurement in situ.

The growing and rapid improvement of smartphone technology (with better cameras and lighting sources) has made these devices increasingly useful in analytical chemistry [19].

In this work, we present the development of reactive supports made of powdered cellulose to determine Cad and Put whose response time is less than $2 \mathrm{~min}$. The signal is measured with a commercial smartphone (using the camera's RGB readout facility), making the methodology fully portable and accessible to any user without specific training.

\section{Material and methods}

\section{Reagents and solutions}

Phosphate buffer solutions $(0.1 \mathrm{M}, \mathrm{pH}$ levels $6.0,7.0$, and 8.0) were prepared from $\mathrm{Na}_{2} \mathrm{HPO}_{4}$ and $\mathrm{NaH}_{2} \mathrm{PO}_{4}$ solids (Sigma S9638 and S9763). Carbonate buffer solution $(0.1 \mathrm{M}$, $\mathrm{pH}$ 9.0) was prepared from $\mathrm{Na}_{2} \mathrm{CO}_{3}$ (Sigma 222321) and $\mathrm{NaHCO}_{3}$ (Sigma S5761).

Hydrogen peroxide stock solution (33\% w/v) was supplied by Panreac (131077.1211). HRP (EC 1.11.1.7) was obtained from Sigma (P8125, 88.6 $\mathrm{U} \mathrm{mg}^{-1}$ ). DAO (EC 1.4.3.22) from Lathyrus cicera $\left(280 \mathrm{U} \mathrm{mL}^{-1}\right)$ was purchased from Molirom P021.

Cadaverine (C8561), putrescine (P7505), histamine (53300), phenylethylamine (P6513), tyramine (T287998), tryptamine (246557), and 3,3',5,5'-tetramethylbenzidine (TMB) (860336) were supplied by Sigma. All solutions were daily weighed and dissolved in the buffer solution (minus TMB, which was dissolved in dimethyl sulfoxide (Panreac 131954.1611). TMB solutions were stored in darkness.

The following chemicals were tested as stabilizers: StabilCoat (SC01-0050), StabilGuard (SG01-0050), StabilZyme Select (SZ03-CF02-00508), and StabilZyme Protein-Free (SZPF-CF01-0050) from SurModics, and sacarose 99\% (102019112) and gelatin 98\% (48723) from Sigma-Aldrich. All of them were solved or diluted in buffer.

Several cellulose types (named as A, B, C, D, and E) were tested. A (Aldrich 310697) and B (Aldrich 11365 Avicel PH101) were microcrystalline cellulose of the size of $20 \mu \mathrm{m}$ and $50 \mu \mathrm{m}$, respectively, and average degree of polymerization of less than 350. C (815050.1, Macherey Nagel \& Co) and D (815060, Macherey Nagel \& Co) were native cellulose of fiber length between 20 and $75 \mu \mathrm{m}$ and 
between 20 and $100 \mu \mathrm{m}$, respectively, and average degree of polymerization of 620-680. E (Sigma 22,184) was cellulose of fiber length between 20 and $250 \mu \mathrm{m}$.

\section{Equipment and instruments}

Molecular absorption measurements were performed using a Hewlett-Packard model HP 8452A diode array spectrophotometer equipped with a HP 89090A Peltier temperature and stirrer control accessory. Depending on the measurement wavelength, quartz (Hellma QS 101) or glass (Hellma Q 101) cuvettes were used.

Cellulose supports were dried into an OVAN incubator (model OM10E).

Two smartphones were used to measure the color development in the cellulose supports: a Nexus $5 \mathrm{X}$ (camera of 12.3 megapixels) and an iPhone 6S (camera of 12 megapixels); with the first smartphone (which work under Android operating system), the application used to capture the RGB coordinates was Color Grab ${ }^{\circledR}$ (from Loomatix), and with the other one (iOS8), the application used was Color Value Pro (V 1.0). Finally, a Nikon D3400 reflex camera was also used. The image file (raw data) was processed with Matlab R2011b (MathWorks, Natick, MA, USA) and ImageJ (version by FIJI, using Java 6) programs for reading RGB. Factorial analysis and data treatments were calculated by both Matlab R2011b and Excel (Microsoft Excel 2010, Redmond, WA, USA).

In this case, an illumination box was used. Indirect constant artificial lighting was produced by a warm white dimmable LED by EGLO (Austria) (7.5 W), which could be selected to red, green, and blue illuminations. Their spectral power densities were characterized in the range 400-700 nm and measured with an arrangement composed by an optical fiber (QP600-1-sR) and a compact monochromator (Ocean Optics QE-65000).

\section{Procedure}

\section{Measurements in solution}

The variation of the absorbance during the enzymatic reaction was initially monitored at $450 \mathrm{~nm}$ and $650 \mathrm{~nm}$ (the diode array spectrophotometer obtains the absorbance in the whole wavelength range from 190 to $1100 \mathrm{~nm}$ ).

To do that, the appropriate concentrations of reagents were added into the cuvette with the buffer solution, giving $2 \mathrm{U} \mathrm{mL}^{-1}$ of DAO, $0.6 \mathrm{U} \mathrm{mL}^{-1}$ of HRP, and $4 \times 10^{-4} \mathrm{M}$ of TMB (total volume in the cell, $2 \mathrm{~mL}$ ). The cuvette was then introduced in the spectrophotometer, the stirrer was connected, and the measurement was started in the kinetic mode. After $50 \mathrm{~s}$ (to obtain the baseline, this period of time can be reduced), $20 \mu \mathrm{L}$ of the analyte (or sample) solution was injected and the variation of the absorbance during the reaction was recorded over the time. As diode array spectrophotometer has reserve optic configuration, a yellow filter must be placed between the lamp $\left(\mathrm{D}_{2}\right)$ and the cell to avoid the photooxidation of the dye. During the optimization studies, the concentration of the reagents and other conditions were modified depending on the parameter to be studied. The maximum absorbance at the chosen wavelength $\left(\mathrm{Abs}_{\max , \lambda}\right)$ was used as the analytical parameter.

\section{Cellulose platform preparation}

The mold used was the lid of a conventional 96-well plate.

Two protocols have been used for preparation of the circular cellulose test supports (CCTSs).

Normal CCTSs On a dispersion of 3\% of cellulose, TMB and HRP were added. Seventy-five microliters of this dispersion $\left(4 \times 10^{-4} \mathrm{M}\right.$ in TMB and $0.6 \mathrm{U} \mathrm{mL}^{-1}$ in HRP) was added into the wells of the mold. The plate was placed into the incubator and dried at $35^{\circ} \mathrm{C}$ for $1 \mathrm{~h}$. Then, $10 \mu \mathrm{L}$ of DAO solution was added and, after $30 \mathrm{~s}, 5 \mu \mathrm{L}$ of the analyte solution (Cad or Put).

Long-life CCTSs On a dispersion of 3\% of cellulose, TMB was added. Seventy-five microliters of this dispersion $\left(4 \times 10^{-4} \mathrm{M}\right.$ in TMB) was put into the wells of the mold plate. The plate is placed into the incubator and dried at $35{ }^{\circ} \mathrm{C}$ for $1 \mathrm{~h}$. Then, $10 \mu \mathrm{L}$ of DAO and HRP was added and, after $30 \mathrm{~s}, 10 \mu \mathrm{L}$ of the stabilizer (usually StabilCoat ${ }^{\mathrm{TM}}$ ). The so-prepared CCTSs are allowed to dry and stored in a closed bag with desiccant in the refrigerator. The analyte determination is carried out by injection of $5 \mu \mathrm{L}$ of the analyte solution (Cad or Put).

Measurements (photographs) were obtained after drying (about $1 \mathrm{~min}$ after sample addition).

For the construction of the test, the supports should be stuck to a polystyrene strip.

\section{Measurements using the smartphone}

To measure the color, the camera of a smartphone and a commercial application, which decomposes the color in RGB value, were used. In order to maintain constant lighting conditions, the position of the smartphone was fixed and the strips (or supports) were moved under it (see Electronic Supplementary Material (ESM) Fig. S1).

Firstly, the RGB zero values were taken before the addition of the analyte (named as $R_{0}, G_{0}$, and $B_{0}$, respectively). Later, the analyte was added and, after $60 \mathrm{~s}$ (time enough to obtain a stable color), the $R, G$, and $B$ values were obtained. The analytical signal was the change in the value of each coordinate $\left(\Delta R\left(R_{0}-R\right)\right.$, $\Delta G\left(G_{0}-G\right)$, and $\left.\Delta B\left(B_{0}-B\right)\right)$ or the relative change

\section{3}

84 85 86 87 88 89 90 1

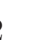
(1) (1) 6 7 9 0 2

3

4

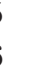

7

8

.

(1)

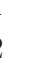

3


observed in the value of each coordinate (sub-index $r$ refers to relative)

$\Delta B, r=\frac{B_{0}-B}{B_{0}} \Delta G, r=\frac{G_{0}-G}{G_{0}} \Delta R, r=\frac{R_{0}-R}{R_{0}}$

\section{Measurements using the digital camera}

The camera was set to automatic focus mode. The size of the picture was adapted according to the size of the CCTSs. The pictures were transferred to a computer using Microsoft Photo Editor $^{\mathrm{TM}}$. RGB values were measured in each image from regions of interest of 1500 pixels in size. The analytical parameters used were as previously indicated in Eq. (1).

\section{$A B$ determination in a tuna sample}

A tuna sample was analyzed by the Laboratorio de Salud Pública of Aragón (LSPA) using a validated method [20]. In brief, $2.50( \pm 0.01) \mathrm{g}$ tuna was weighted in a flask together with $100 \mu \mathrm{L}$ histamine dihydrochloride-d4 (internal standard, $100 \mathrm{mg} \mathrm{L}^{-1}$ ) and $20 \mathrm{~mL}$ of $5 \%$ trichloroacetic acid. The mixture was first manually shaken during $30 \mathrm{~s}$, then submitted to ultrasounds $(10 \mathrm{~min})$ and finally centrifuged $\left(4{ }^{\circ} \mathrm{C}\right)$ at $4000 \mathrm{rpm}$ during $10 \mathrm{~min}$. The solution was filtered (filter paper) and taken to $50 \mathrm{~mL}$ with $5 \%$ trichloroacetic acid. Ten microliters of this solution were injected in a HPLC-MS instrument. The following concentrations were obtained (in $\mathrm{mg} \mathrm{kg}^{-1}$ ): $100 \pm 11$ Put, $380 \pm 19 \mathrm{Cad}, 900 \pm 40 \mathrm{His}, 300 \pm$ $22 \mathrm{Tyr}, 20.0 \pm 2.2$ tryptamine, and $50.0 \pm 5.6$ phenylethylamine. This sample was also analyzed by the method presented in this paper; the sample treatment used was similar to that described except the addition of the internal standard.

\section{Results and discussion}

\section{Studies in solution}

\section{Development of the method: analytical figures of merit for Put and Cad}

As mentioned in the "Introduction," the analytical system developed in this study is based on a sequence of enzymatic reactions (Scheme 1) which results in the appearance of a blue color. Although the TMB/HRP/ $\mathrm{H}_{2} \mathrm{O}_{2}$ indicating reaction has been extensively studied, there are two important aspects which have not yet been fully clarified regarding TMB and HRP concentrations: (1) A concentration ratio of TMB to $\mathrm{H}_{2} \mathrm{O}_{2}$ formed of 10:1 or higher should be used to obtain the maximum stable signal (see ESM Fig. S2), and (2) many authors have indicated that the blue color of the $\mathrm{TMB}_{\mathrm{ox}}$ species disappears over time. In this study, we have demonstrated that HRP is able to regenerate (in part) TMB from $\mathrm{TMB}_{\mathrm{ox}}$ (see ESM Fig. S3). The HRP concentration is critical, with a maximum obtained using $0.60 \mathrm{U} \mathrm{mL}^{-1}$.

There are additional interesting aspects of the optimization of Put and Cad determination: (1) It was observed that the DAO concentration (ESM Fig. S4) affects the kinetic of the reaction but does not affect the maximum signal obtained or its stability. With $2 \mathrm{U} \mathrm{mL}^{-1}$, the maximum signal was obtained in less than 2 min. (2) Both the formation of the blue TMB compound and the activity of both enzymes depend on the $\mathrm{pH}$. Therefore, the effect of this parameter on the global yield was studied. ESM Fig. S5 shows the results obtained. As can be seen, with $\mathrm{pH} 6$, the signal is at a maximum and stable, although the reaction time is longer.

The analytical figures of merit for Put and Cad determination have been obtained using the absorbance change at $650 \mathrm{~nm}$ as the analytical parameter. The linear response range (ESM Fig. S6), the sensitivity, and the RSD for both BAs are shown in Table 1. As can be seen, both analytes can be determined with the same sensitivity ( $t$ test) and the linear response range obtained allows the application of the method to real samples. This table also shows the calibration line obtained using only $\mathrm{H}_{2} \mathrm{O}_{2}$. The slope of the calibration line is slightly higher than that obtained for Put and Cad; this can be explained considering that, like HRP, DAO is also able to partially reduce $\mathrm{TMB}_{\mathrm{ox}}$ to $\mathrm{TMB}$, which is demonstrated when the $\mathrm{H}_{2} \mathrm{O}_{2} / \mathrm{TMB} / \mathrm{HRP}$ reaction is carried out in the presence of DAO (see ESM Fig. S7).

\section{Study of interferences: His determination}

Many food samples containing Put and Cad also contain other biogenic amines, especially His and Tyr. The DAO used in this work presents a specificity $\left(k_{\text {cat }} / K_{\mathrm{M}}\right)$ [21] for Put and Cad which is about 100 times higher than that for Tyr or His. Nevertheless, an interference study was carried out. The results show that His and Tyr do not interfere until, at least, a His (Tyr):Put (Cad) ratio of 10:1 is obtained. Tyr did not modify either the $\mathrm{Abs}_{\max }$ or the $\mathrm{Abs}=f(t)$ profiles obtained with Put/ Cad. However, the effect of His was different (Fig. 1). This compound did not affect the $\mathrm{Abs}_{\text {max }}$, but it produced a later gradual decrease in the absorbance value obtained. Different tests (compiled in ESM Fig. S8) were performed in order to elucidate the mechanism of this negative slope. The conclusion was that the aldehyde derived from the His (imidazole acetaldehyde) is able to regenerate $\mathrm{TMB}_{\mathrm{ox}}$, reducing its absorbance. As can be seen, the kinetic of this reaction is not very fast so the decrease in the absorbance follows a straight line. Interestingly, the slopes of these decreases proportionally change with the His concentration (see Fig. 1, inset), but the His concentrations need to be high in order to detect this effect. This effect could be used for the simultaneous 
Smartphone-interrogated test supports for the enzymatic determination of putrescine and cadaverine in food

t1.1 Table 1 Calibration line in the

t1.2 solution of cadaverine, putrescine, and hydrogen

t1.3 peroxide

t1.4

\begin{tabular}{llll}
\hline & Calibration line $([\mathrm{Cad}]$ or $[\mathrm{Put}]$ in $\mathrm{M})$ & Range $(\mathrm{M})$ & RSD, \% $(n)$ \\
\hline Cadaverine & $\mathrm{Abs}=29,502[\mathrm{Cad}]+0.001 ; r=0.999$ & $1.0 \times 10^{-6}$ to $1 \times 10^{-5 *}$ & $1.8(5)$ \\
Putrescine & $\mathrm{Abs}=29,740[\mathrm{Put}]+0.001 ; r=0.999$ & $1.0 \times 10^{-6}$ to $1 \times 10^{-5 *}$ & $0.8(5)$ \\
Peroxide & $\mathrm{Abs}=38,788\left[\mathrm{H}_{2} \mathrm{O}_{2}\right]+0.0001 ; r=0.999$ & $5.0 \times 10^{-7}$ to $6 \times 10^{-6}$ & $2.3(5)$ \\
\hline
\end{tabular}

Conditions: $\mathrm{HRP}=0.6 \mathrm{U} \mathrm{mL}^{-1}, \mathrm{TMB}=1 \times 10^{-4} \mathrm{M}, \mathrm{pH}=6, \mathrm{DAO}=1.8 \mathrm{U} \mathrm{mL}^{-1}, \mathrm{pH}=6, \lambda=650 \mathrm{~nm}$

*Maximum concentration tested determination of Put/Cad and His in samples containing very high His concentrations compared with those of Put/Cad.

\section{Put and Cad determination in a tuna sample}

The method was applied to Put + Cad determination in the previously described (section " $\mathrm{AB}$ determination in a tuna sample") tuna sample. Note that the His and Tyr concentrations present in the sample are below the interference limit. The result obtained was $490( \pm 40) \mathrm{mg} \mathrm{kg}^{-1}(n=3)$, which is statistically similar to that obtained by the HPLC-MS reference method ( $95 \%$ confidence $t_{\text {cal }}=0.4<t_{\text {crit }}=2.78$ ). This validates the described procedure.

\section{Measurement system optimization}

\section{Mathematical description}

The aim of this study is to prepare test supports for the rapid determination of Put/Cad. This involves the use of a smartphone or a digital camera as the instrument and RGB coordinates as the analytical signals. In this section, we are interested in obtaining a mathematical relationship between the $R, G$, and $B$ responses given by the instrument to the three color coordinates $\left(E_{(R, G, B)}\right)$ and the absorbing species concentration $\left(C, \mathrm{mg} \mathrm{L}^{-1}\right)$, in this case $\mathrm{TMB}_{\mathrm{ox}}$. These responses are given by [22]

$E_{(R, G, B)}=A \sum_{\lambda} I_{\lambda} P_{\lambda} R_{\lambda}$

where $A$ is a constant including constant factors related with the camera design, the solid angle, the light-to-voltage transformation, and the analog-to-digital conversion; $I_{\lambda}$ is the spectral power of the illumination source which is usually known and independent of the characteristics of the solid to be measured; $P_{\lambda}$ is the spectral sensitivity of the camera (this parameter is characteristic of each smartphone or camera); and $R_{\lambda}$ is the reflectance of the solid (test strips, in this case). This parameter depends on the absorbing species present in the solid $\left(C\right.$, in $\mathrm{M}$, and $\varepsilon_{\lambda}$ in $\left.\mathrm{M}^{-1} \mathrm{~cm}^{-1}\right)$ and the scattering coefficient of the solid $\left(s_{\lambda}\right.$, in $\left.\mathrm{cm}\right)$. Several models have been proposed to relate the $R_{\lambda}$ with $a_{\lambda}, s_{\lambda}$, and $C$. The most commonly used is that derives from the Kubelka-Munk theory adapted to TLC plate measurements [23]

Fig. 1 Effect of histamine in the putrescine signal. Experimental conditions: $[\mathrm{TMB}]=6 \times 10^{-5} \mathrm{M}$, $[\mathrm{HRP}]=0.5 \mathrm{Um} \mathrm{L}^{-1},[\mathrm{DAO}]=$ $1 \mathrm{U} \mathrm{mL}^{-1},[$ Put $]=2 \times 10^{-6} \mathrm{M}$, $\lambda=650 \mathrm{~nm}$

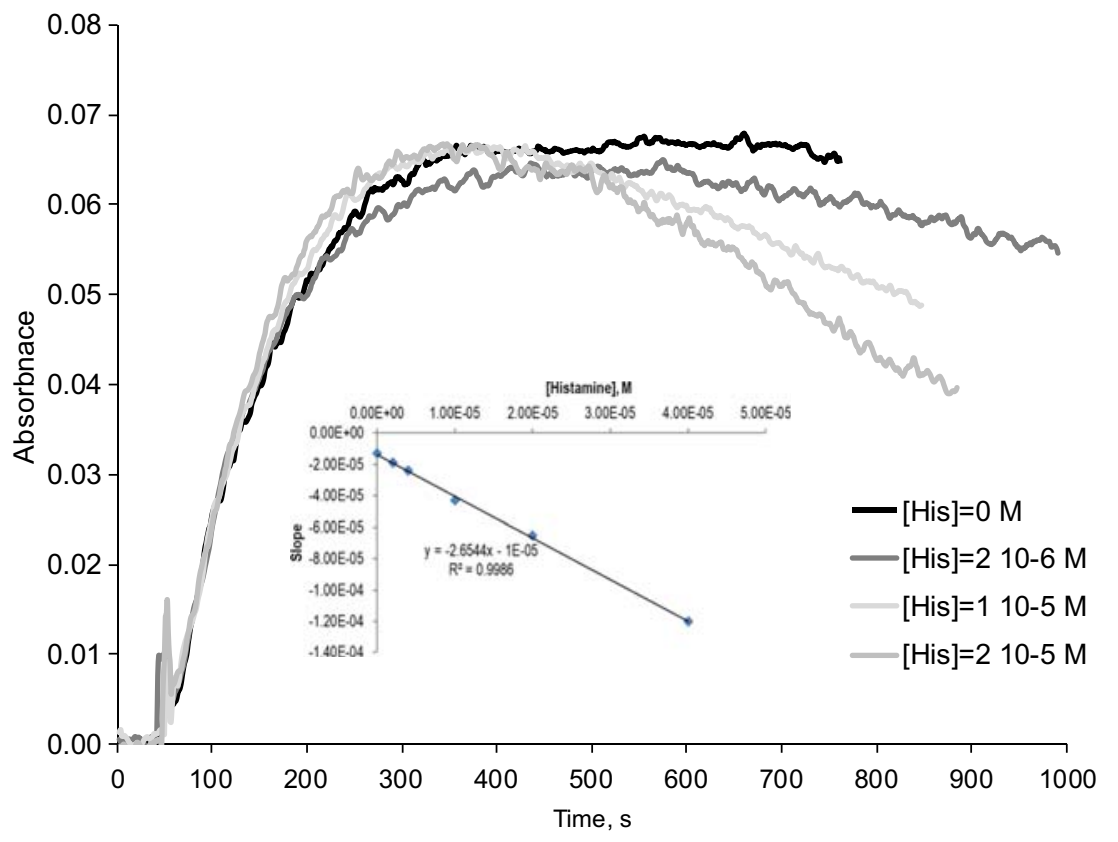




$$
\begin{aligned}
R_{\lambda} & =\frac{1}{\alpha_{\lambda}+\beta_{\lambda} \operatorname{Coth}\left(\beta_{\lambda} s_{\lambda} L\right)} \alpha_{\lambda}=\frac{2,3 \varepsilon_{\lambda} C+s_{\lambda}}{s_{\lambda}} \beta_{\lambda} \\
& =\sqrt{\alpha^{2}-1}
\end{aligned}
$$

361

363 where Coth is the hyperbolic cotangent and $L$ is the optical pathlength. A combination of Eqs. (2) and (3) gives

$$
E_{(R, G, B)}=A \sum_{\lambda} I_{\lambda} P_{\lambda} K_{\theta} \frac{1}{\alpha_{\lambda}+\beta_{\lambda} \operatorname{Coth}\left(\beta_{\lambda} s_{\lambda} L\right)}
$$

Equation (4) suggests a complex relationship between $E_{(R, G, B)}$ and the analyte concentration. However, this can be simplified by applying a polynomial Taylor series approximation, yielding (see ESM Appendix 1)

$E_{(R, G, B)}=A E_{0,(R, G, B)}+A \sum_{i} \frac{1}{i} E_{i,(R, G, B)} c^{i}$

where $E_{0,(R, G, B)}$ is the response obtained when the concentration of the absorbing species is zero

$E_{0,(R, G, B)}=A \sum_{\lambda} I_{\lambda} P_{\lambda}\left(\frac{s_{\lambda} L}{1+s_{\lambda} L}\right)$

As it is well known, the minimum order of the polynomial depends on the width of the concentration range to be covered and the complexity of the original function. In this case, $i=2$ gives a suitable balance between the concentration range and the robustness of the calibration line. As indicated in the ESM

$E_{1,(R, G, B)}=-\sum_{\lambda} I_{\lambda} P_{\lambda}\left[\left(\frac{s_{\lambda} L}{1+s_{\lambda} L}\right)^{2}\left[\frac{3+2 s_{\lambda} L}{3 s_{\lambda}}\right]\right] 2,3 \varepsilon_{\lambda} c(7)$

$E_{2,(R, G, B)}=\sum_{\lambda} I_{\lambda} P_{\lambda}\left[\left(\frac{s_{\lambda} L}{1+s_{\lambda} L}\right)^{3}\left[\frac{30+45 s_{\lambda} L+24\left(s_{\lambda} L\right)^{2}+4\left(s_{\lambda} L\right)^{3}}{45 s_{\lambda}^{2}}\right]\right]\left(2,3 \varepsilon_{\lambda} c\right)^{2}$

To avoid the effect of the stray light and $A$ in the analytical signal, $\Delta E$ and $\Delta E_{r}$ were used

$$
\begin{aligned}
& \Delta E_{(R, G, B)}=E_{0,(R, G, B)}-E_{(R, G, B)} \\
& \Delta E_{(R, G, B), r}=\frac{E_{0,(R, G, B)}-E_{(R, G, B)}}{E_{0,(R, G, B)}}
\end{aligned}
$$

\section{Adaptation of $\mathrm{H}_{2} \mathrm{O}_{2}$ commercial test strips for $\mathrm{BA}$ sensing}

Commercial strips for peroxides are commercially available (Quantofix Peroxide $25^{\circledR}$ ). As far as we know, these strips contain HRP and a colorant similar to TMB (the concentrations are unknown). The possibility of using these strips to detect Put and Cad was studied. In order to do so, $5 \mu \mathrm{L}$ of a $50 \mathrm{U} \mathrm{mL}^{-1}$ DAO solution was added to each strip and allowed to dry for $1 \mathrm{~min}$. Then, $5 \mu \mathrm{L}$ of Put of concentrations between 1.0 and $65 \mathrm{mg} \mathrm{L}^{-1}$ (equivalent to the 0.5 to $25 \mathrm{mg} \mathrm{L}^{-1} \mathrm{H}_{2} \mathrm{O}_{2}$ range for which the strips were designed) was added and the reaction was allowed to develop for $30 \mathrm{~s}$. The $\Delta E_{R, r}, \Delta E_{G, r}$, and $\Delta E_{B, r}$ values were obtained; the results are compiled in ESM Fig. S9. Some interesting conclusions can be obtained from these results: (1) The highest sensitivity provided by the component $R\left(\Delta E_{R}, r\right)$ could be expected when the molecular absorption spectra of the $\mathrm{TMB}_{\mathrm{ox}}$ are compared with the spectral responsivity of the smartphone (ESM Fig. S10). As can be seen, $\mathrm{TMB}_{\mathrm{ox}}$ (which is blue in color) shows very little absorbance at the $B$-component wavelength range, a little more at the $G$-component, and high absorbance at the $R$-component wavelength ranges. Interestingly, these three filters also transmit some light in the NIR region (between 800 and $1000 \mathrm{~nm}$ ). Since the $\mathrm{TMB}_{\mathrm{ox}}$ presents an additional absorption band in this spectral zone (centered at $890 \mathrm{~nm}$ ), the signal is finally observed working with $E_{B}$. (2) Focusing on the $E_{R}$ values, a linear response is observed for low Put concentrations (ESM Fig. S9B), but a second-order polynomial response better fits the experimental results for a wider concentration range (ESM Fig. S9A). (3) The results indicate that it is possible to use this type of commercial test strip for semi-quantitative Put and Cad determination after adding DAO.

\section{Instrument optimization}

As has been indicated, $P_{\lambda}$ depends on the smartphone or camera used. Since this parameter appears in the coefficients of the calibration line $\left(E_{1(R, G, B)}\right.$ and $\left.E_{2(R, G, B)}\right)$, different sensitivity levels are expected to be obtained with different smartphones or cameras. In this study, we used two different smartphones (iPhone 6S and Nexus QQ) and a digital camera. The $\Delta E_{R, r}$ results obtained using the commercial test strips modified with DAO for Put determination are shown in Fig. 2.

\section{Optimization of circular test supports}

\section{Support design}

Although the toxicity of biogenic amines is not questioned, the level of toxicity is difficult to establish because it depends on the characteristics of each person and the level of other amines. There is no legislation, but a maximum level of total BA of 750-900 $\mathrm{mg} \mathrm{kg}^{-1}$ has been suggested [1]. The European Union has established regulations only for His. Its level should be below $100 \mathrm{mg} \mathrm{kg}^{-1}$ in raw fish and below $200 \mathrm{mg} \mathrm{kg}^{-1}$ in salted fish for species belonging to the Scombridae and Clupeidae families (Commission Regulation (EC) 2073/2005). Since there is no legislation for the other BAs, in this work, a concentration of $100 \mathrm{mg} \mathrm{kg}^{-1}$ was chosen as a reference to prepare the test supports sensitive to Put and Cad. The CCTSs were initially synthesized following the procedure "Normal CCTSs" described in section "Cellulose platform preparation." 
Fig. 2 Sensitivity comparison between two smartphones and a digital camera. Experimental conditions: commercial strip test of $\mathrm{H}_{2} \mathrm{O}_{2}$ (Quantofix Peroxide $25^{\circledR}$ ) plus $5 \mu \mathrm{L}$ of $50 \mathrm{U} \mathrm{mL}^{-1}$ DAO solution

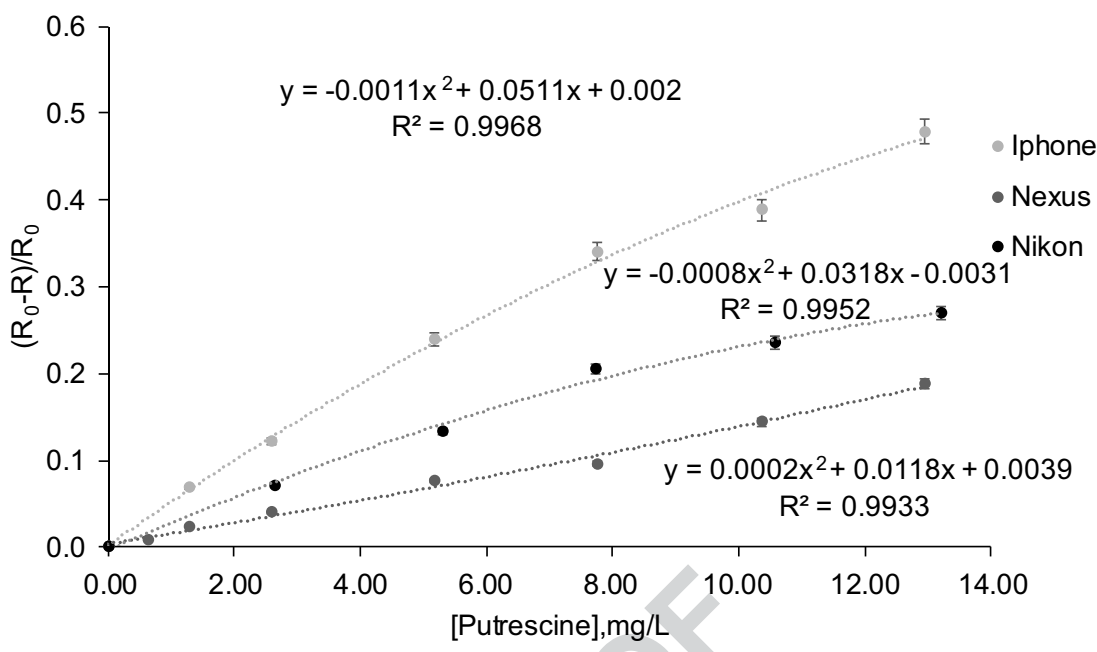

Different supports were used for obtaining the sensing platforms; two were finally chosen. Most of the optimizations were carried out using the lid of a plastic 96-well plate whose diameter (around $0.5 \mathrm{~cm}$ ) was suitable for obtaining CCTSs to be measured with the smartphone.

Larger CCTSs (ESM Fig. S11), which allow the calibration and determination to be performed in the same support, were also tested with good results.

To design a quick, sensitive, and reproducible method, the kinetic of the reaction should be fast, and the color produced during the reaction should be as intense and homogenous as possible. To obtain these properties, two aspects need to be controlled: (a) The analyte should diffuse in a controlled way through the CCTSs, avoiding undesirable phenomena such as the "coffee ring effect" (the product of the reaction is accumulated in the outside area), and (b) the enzyme should not lose activity after immobilization. Both depend on the material used (type of cellulose and the concentration and the volume of the cellulose solution used for obtaining CCTSs) and the way in which the enzyme is immobilized. Several types of cellulose were tested, but only the 5 cited in section "Reagents and solutions" were able to give robust CCTSs. Figure S12 (see the ESM) shows the signal obtained using different concentrations ranging from 1 to $5 \%$ of the different types of cellulose chosen (less than $1 \%$ gives a soft CCTS, and higher than $5 \%$ did not provide enough reproducibility). Three percent was finally chosen balancing sensitivity, reaction time, and easy handling. All the celluloses gave good results
(Table 2), but cellulose A gave a better-defined spot; the signal was significantly higher (1-factor ANOVA, 95\%).

Regarding the sample volume to be injected, $50 \mu \mathrm{L}, 75 \mu \mathrm{L}$, and $100 \mu \mathrm{L}$ were tested. As can be seen in ESM Table S1, there was no influence on the signal. However, in terms of ease of use, the best results were found using $75 \mu \mathrm{L}$.

Enzyme immobilization by entrapment is desirable to obtain a homogeneous color, but adsorption avoids loss of activity. The results obtained in the previous study indicated that HRP can be entrapped. However, DAO entrapment gives very small signals and a slower reaction rate (see ESM Fig. S12), so it was decided to immobilize DAO by adsorption (see section "Reagents and solutions").

\section{Optimization of experimental conditions}

The quantity of immobilized reagents affects the sensitivity and the kinetic of the enzymatic reactions. It also affects the selectivity, if the amines compete for the DAO. The results obtained for the optimization of DAO (Fig. 3) show that above 0.25 unit of DAO/CCTS, the $\Delta E_{R}$ does not depend on the DAO amount. The response time decreases as the amount of DAO increases; 0.25 unit $\mathrm{CCTS}^{-1}$ was chosen as the appropriate quantity since the signal is at a maximum, the response time is suitable for a fast method ( $30 \mathrm{~s}$ until a stable signal is achieved), and the amount of the reagent consumed is reasonable.
Table 2 Signal obtained $(\Delta R)$ with different types of cellulose

\begin{tabular}{|c|c|c|c|c|c|}
\hline & \multicolumn{5}{|l|}{ Cellulose } \\
\hline & A & $\mathrm{B}$ & $\mathrm{C}$ & $\mathrm{D}$ & $\mathrm{E}$ \\
\hline$\Delta R \pm \sigma$ & $56.67 \pm 2.0$ & $51.67 \pm 1.9$ & $46.00 \pm 1.5$ & $49.00 \pm 1.6$ & $48.67 \pm 1.6$ \\
\hline
\end{tabular}

Conditions: $3 \%$ cellulose, $[\mathrm{HRP}]=1.0 \mathrm{U} \mathrm{mL}^{-1},[\mathrm{TMB}]=6 \times 10^{-4} \mathrm{M}$ and $\mathrm{pH}=7$ in the cocktail of reagents, $\mathrm{DAO}=0.25 \mathrm{U} \mathrm{CCTS}^{-1}$ before drying, $[\mathrm{Cad}]=1 \times 10^{-4} \mathrm{M}, n=3$ 
Fig. 3 Optimization of DAO quantity. Experimental conditions: manufacturing procedure described in "Normal CCTSs" under section "Cellulose platform preparation" (3\% cellulose, $[\mathrm{HRP}]=0.5 \mathrm{U} \mathrm{mL}^{-1}$, $[\mathrm{TMB}]=6 \times 10^{-4} \mathrm{M},[\mathrm{Cad}]=$ $1 \times 10^{-4} \mathrm{M}, \mathrm{pH}=7.0$ )

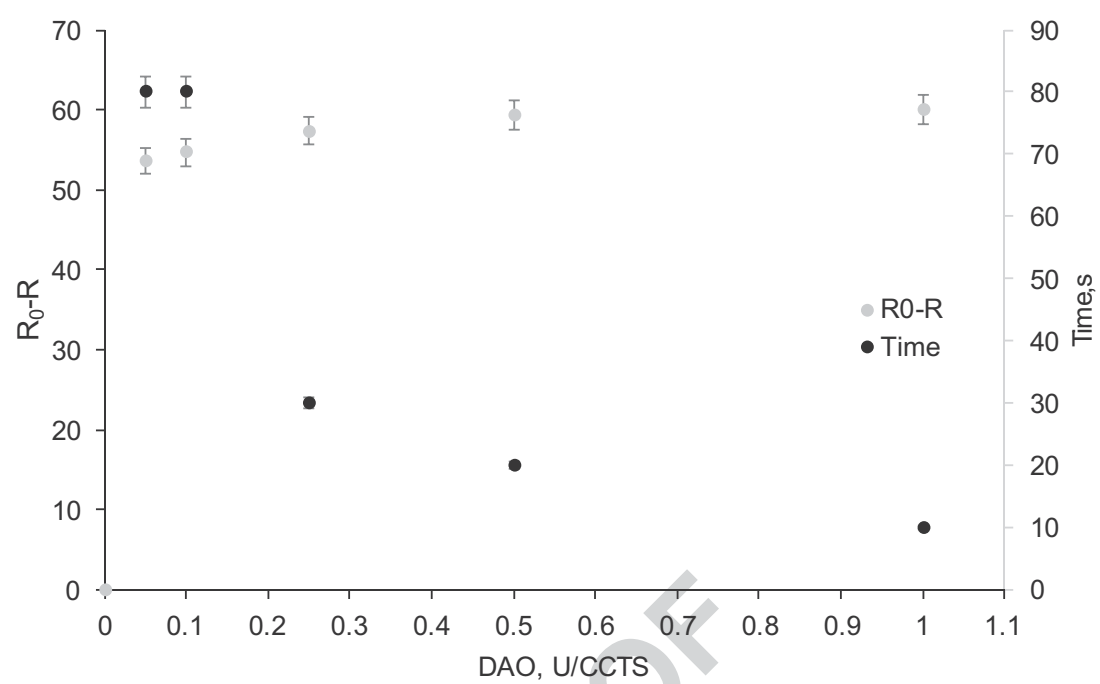

Table S2 (see the ESM) shows that the best results were obtained working with a TMB:Put molar ratio of 10:1 or higher in the CCTSs. The 1-factor ANOVA showed that there are no significant differences for molar ratios higher than 10 . This agrees with the results obtained in solution. Finally, the HRP (ESM Fig. S13) and pH (ESM Table S3) were also optimized.

A very important parameter to be considered is the CCTSs' life time. Under the optimal conditions chosen, the lifetime was only 4 days. Therefore, the following chemicals were tested in order to improve the stability of the enzymes: StabilCoat, StabilGuard, StabilZyme/HRP, StabilZyme Select, trehalose, sacarose, and gelatin. A very exhaustive study was carried out including the cellulose concentration and the optimum moment of the CCTS synthesis at which the stabilizer and enzymes should be added (ESM Appendix 2).
The best results were found using StabilCoat and StabilGuard, but the manufacturing procedure used was changed to the "Long-life CCTSs" procedure described in section "Cellulose platform preparation." In these conditions, the lifetime of the platform is extended to at least 45 days (Fig. 4). The maximum signal is not obtained immediately (ESM Fig. S12). As can be seen, at $30 \mathrm{~s}$, the maximum signal is obtained working with $3 \%$ cellulose. The signal is stable for at least $5 \mathrm{~min}$.

\section{Analytical characteristics. cadaverine and putrescine determination in tuna}

The analytical figures of merit were obtained in the optimized conditions. The response range (ESM Fig. S14), the sensitivity, and the RSD for both BAs are shown in Table 3.
Fig. 4 Effect of stabilizers on the lifetime of the CCTSs.

Experimental conditions: manufacturing procedure described in "Long-life CCTSs" under section "Cellulose platform preparation" ( $3 \%$ cellulose, $[\mathrm{TMB}]=6 \times 10^{-4} \mathrm{M},[\mathrm{HRP}]=$ $0.045 \mathrm{U} \mathrm{CCTS}^{-1},[\mathrm{DAO}]=$ $0.2 \mathrm{U} \mathrm{CCTS}^{-1}$, [putrescine] $=$ $\left.2 \times 10^{-4} \mathrm{M}, \mathrm{pH}=7, n=3\right)$. SGIS, StabilGuard; SCIS, StabilCoat; SZSS, StabilZyme Select; SZPF, StabilZyme Protein-Free

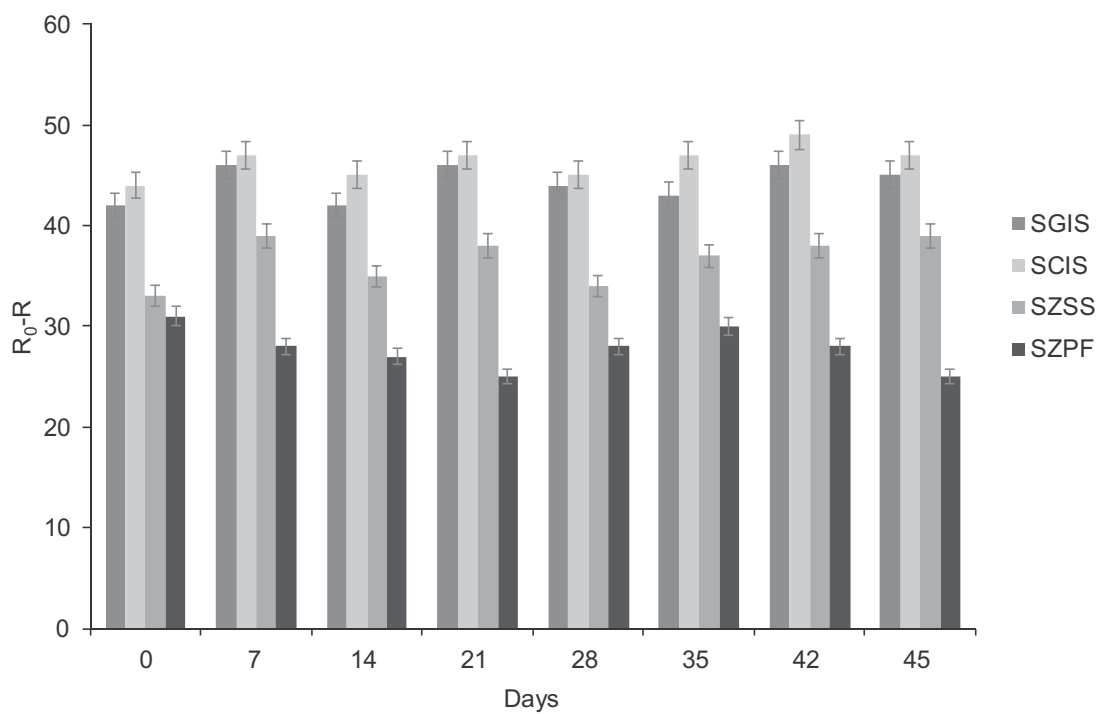


Smartphone-interrogated test supports for the enzymatic determination of putrescine and cadaverine in food

t3.1

$\mathrm{t} 3.2$

t3.3 Cadaverine

$\mathrm{t} 3.4$

t3.5 Putrescine

$\mathrm{t} 3.6$

$\mathrm{t} 3.7$

Table 3 Calibration line in CCTSs of cadaverine, putrescine, and hydrogen peroxide

\begin{tabular}{llll}
\hline & Calibration line $([\mathrm{Cad}]$ or $[\mathrm{Put}]$ in $\mathrm{M})$ & Range $(\mathrm{M})$ & $\mathrm{RSD}, \%(n)$ \\
\hline Cadaverine & $\left(R_{0}-R\right) / R_{0}=1785[\mathrm{Cad}]-0.0004 ; r=0.999$ & $5.0 \times 10^{-5}$ to $2 \times 10^{-4}$ & $3.2(4)$ \\
& $\left(R_{0}-R\right) / R_{0}=-576,792[\mathrm{Cad}]^{2}+1906[\mathrm{Cad}]-0.004 ; r=0.999$ & $5.0 \times 10^{-5}$ to $4 \times 10^{-4 *}$ & $3.2(4)$ \\
Putrescine & $\left(R_{0}-R\right) / R_{0}=1844[\mathrm{Put}]+0.0042 ; r=0.999$ & $5.0 \times 10^{-5}$ to $2 \times 10^{-4}$ & $3.1(4)$ \\
& $\left(R_{0}-R\right) / R_{0}=-965,171[\mathrm{Put}]^{2}+2039[\mathrm{Put}]-0.001 ; r=0.999$ & $5.0 \times 10^{-5}$ to $4 \times 10^{-4 *}$ & $3.1(4)$ \\
Peroxide & $\left(R_{0}-R\right) / R_{0}=2211\left[\mathrm{H}_{2} \mathrm{O}_{2}\right]+0.004 ; r=0.997$ & $1.5 \times 10^{-5}$ to $3 \times 10^{-4}$ & $3.8(4)$ \\
\hline
\end{tabular}

Conditions: $3 \%$ cellulose, $[\mathrm{HRP}]=0.5 \mathrm{U} \mathrm{mL}^{-1},[\mathrm{TMB}]=6 \times 10^{-4} \mathrm{M}$ and $\mathrm{pH}=7$ in the cocktail of reagents, DAO $=0.5 \mathrm{UCCTS}^{-1}$

*Maximum concentration tested

For both BAs, a linear range was obtained up to $2 \times$ $10^{-4} \mathrm{M}$, with the lowest concentration tested being $2 \times$ $10^{-5} \mathrm{M}$, which would allow the determination in real samples. A second-degree polynomial response allows to extend the response range up to concentration values of at least $5 \times$ $10^{-4} \mathrm{M}$. Both analytes present the same sensitivity in CCTSs. To demonstrate the capability of the method for the Put + Cad joint determination, an additional study was carried out with mixtures of Put and Cad between 1:1 and 1:4 (or vice versa). The signals obtained for these solutions were interpolated in both the calibration lines (Put and Cad). The errors obtained were between 3 and 6\% (ESM Table S4), which would allow the determination of the total concentrations of cadaverine and putrescine in real samples.

As it occurs in solution, the slope of $\mathrm{H}_{2} \mathrm{O}_{2}$ calibration line (measured using CCTSs) is higher than the Put and Cad slopes (Table 3).

The effect produced by Tyr and His was also studied. His does not produce any interference in the Put/Cad signal at least up to a 5:1 M ratio (ESM Fig. S15a). However, if the spots are observed after a 5-min reaction (ESM Fig. S15b), the $\mathrm{TMB}_{\mathrm{ox}}$ signal has disappeared due to a similar effect to that produced in the solution. This shows that His is present in the sample, even in the case of 2:1 ratio, for which histamine does not interfere. Tyr does not produce any interference in the Put/ Cad signal at least up to 10:1 $\mathrm{M}$ ratio.

Put and Cad present in the extract of the tuna sample (see section "Put and Cad determination in a tuna sample") were analyzed using the CCTSs designed here. The result obtained was $460 \pm 65 \mathrm{mg} \mathrm{kg}^{-1}(n=3)$. This result is statistically similar, according to a $t$ test $\left(95 \%\right.$ confidence $\left.t_{\text {cal }}=0.51<t_{\text {crit }}=2.78\right)$, to that obtained for the sample using the HPLC-MS method. This enables us to validate this method for the determination of $\mathrm{Cad}+$ Put in the presence of other biogenic amines such as histamine and tyramine in high concentrations in this type of sample.

\section{Conclusions}

It has been demonstrated that long-life CCTSs based on the combined enzymatic reactions given in Scheme 1 can be easily synthesized for Put + Cad joint determination in tuna, both semi-quantitative with the naked eye and quantitative by measuring the $R$ color coordinate with a smartphone and a commercial application. Since the $R$ coordinate has been related to the concentration of BAs, a quantitative method can be considered. The minimum detectable concentration is $2 \times$ $10^{-5} \mathrm{M}$ which, following the sample treatment proposed, enables biogenic amine levels to be quantitatively measured below $100 \mathrm{mg} \mathrm{kg}^{-1}$, which can be considered the critical safety level. In this case, the method would serve as a quality and freshness evaluation technique. This, together with the short response time, the ease of handling, and the possibility of measuring with a smartphone device, makes these supports a good option for the in situ determination of biogenic amines in food.

Acknowledgments $\mathrm{J}$ Navarro thanks to the Government of Aragon (DGA) for a grant. The authors are also very grateful to Dr. Francisco Palacios and MSc Cristina Asensio from Laboratorio de Salud Pública de Aragón (LSPA, DGA) for their invaluable help. This work was the winner of a Poster Prize awarded for excellent presentation of particularly significant innovative analytical research at X CIE/XXVI RNE. This award was sponsored by Analytical and Bioanalytical Chemistry.

Funding information This work was supported by the MINECO of Spain (project CTQ2016-76846R) and by research groups funding DGAFEDER (group E25_17R).

\section{Compliance with ethical standards}

Conflict of interest The authors declare that they have no conflict of interest.

Human and animal interest The research does not involve human participants and/or animals.

\section{References}

1. Ladero V, Calles-Enríquez M, Fernández M, Álvarez MA. Toxicological effects of dietary biogenic amines. Curr Nutr Food Sci. $2010 ; 6: 145-56$. https://doi.org/10.2174/ 157340110791233256.

2. Bulushi IA, Poole S, Deeth HC, Dykes GA. Biogenic amines in fish: roles in intoxication, spoilage, and nitrosamine formation-a 
review. Crit Rev Food Sci Nutr. 2009;49:369-77. https:/doi.org/ 10.1080/10408390802067514.

3. Choi MH, Kim KR, Kim YT, Chung BC. Increased polyamine concentrations in the hair of cancer patients. Clin Chem. 2001;47: 143-4. https://doi.org/10.1093/clinchem/47.1.143.

4. Ernestus RI, Röhn G, Schröder R, Els T, Klekner Á, Paschen E, et al. Polyamine metabolism in brain tumours: diagnostic relevance of quantitative biochemistry. J Neurol Neurosurg Psychiatry. 2001;71:88-92. https://doi.org/10.1136/jnnp.71.1.88.

5. Levêque J, Foucher F, Bansard JY, Havouis R, Grall JY, Moluinoux JP. Polyamine profiles in tumor, normal tissue of the homologous breast, blood, and urine of breast cancer sufferers. Breast Cancer Res Treat. 2000;60:99-105. https://doi.org/10.1023/A: 1006319818530

6. Önal A, Tekkeli SEK, Önal C. A review of the liquid chromatographic methods for the determination of biogenic amines in foods. Food Chem. 2013;138:509-15. https://doi.org/10.1016/j. foodchem.2012.10.056.

7. Erim FB. Recent analytical approaches to the analysis of biogenic amines in food samples. Trends Anal Chem. 2013;52:239-47. https://doi.org/10.1016/j.trac.2013.05.018.

8. Ordóñez JL, Troncoso AM, García-Parrilla MDC, Callejón RM. Recent trends in the determination of biogenic amines in fermented beverages - a review. Anal Chim Acta. 2016;939:10-25. https://doi. org/10.1016/j.aca.2016.07.045.

9. Lerke PA, Porcuna MH, Hin HB. Screening test for histamine in fish. J Food Sci. 1983;48:155-7. https://doi.org/10.1111/j.13652621.1983.tb14812.x

10. Tombelli S, Mascini M. Electrochemical biosensors for biogenic amines: a comparison between different approaches. Anal Chim Acta. 1998;358:277-84. https://doi.org/10.1016/S0003-2670(97) 00606-5.

11. Leonardo $\mathrm{S}, \mathrm{Campàs} \mathrm{M}$. Electrochemical enzyme sensor arrays for the detection of the biogenic amines histamine, putrescine and cadaverine using magnetic beads as immobilisation supports. Microchim Acta. 2016;183:1881-90. https://doi.org/10.1007/ s00604-016-1821-8.

12. Yang X, Feng B, He X, Li F, Ding Y, Fei J. Carbon nanomaterial based electrochemical sensors for biogenic amines. Microchim Acta. 2013;180:935-56. https://doi.org/10.1007/s00604-0131015-6.

13. Kivirand K, Rinken T. Biosensors for biogenic amines: the present state of art mini-review. Anal Lett. 2011;44:2821-33. https://doi. org/10.1080/00032719.2011.565445.

14. Lee JI, Jang JH, Yu MJ, Kim YW. Construction of a bifunctional enzyme fusion for the combined determination of biogenic amines in foods. J Agric Food Chem. 2013;61:9118-24. https://doi.org/10. $1021 / \mathrm{jf} 403044 \mathrm{~m}$.

15. Navarro J, Sanz-Vicente I, Lozano R, de Marcos S, Galbán J. Analytical possibilities of putrescine and cadaverine enzymatic colorimetric determination in tuna based on diamine oxidase: a critical study of the use of ABTS. Talanta. 2020;208:120392. https://doi. org/10.1016/j.talanta.2019.120392.

16. Chen YL, Jonathan Y. Enzyme immobilization on cellulose matrixes. J Bioact Compat Polym. 2016:1-15. https://doi.org/10.1177/ 0883911516637377.

17. Hall M, Eldridge DB, Saunders RD, Fairclough DL, Bateman RC. A rapid dipstick test for histamine in tuna. Food Biotechnol. 1995;9:39-57. https://doi.org/10.1080/08905439509549884.

18. Hall M, Sykes PA, Fairclough DL, Lucchese J, Rogers P, Staruszkiewicz W, et al. A test strip for diamines in tuna. JAOAC Int. 1999;82:1102-8.

19. Calabria D, Caliceti C, Zangheri M, Mirasoli M, Simoni P, Roda A. Smartphone-based enzymatic biosensor for oral fluid L-lactate detection in one minute using confined multilayer paper reflectometry.
Biosens Bioelectron. 2017;94:124-30. https://doi.org/10.1016/j. bios.2017.02.053.

20. ITE-FQ080-02: determination of biogenic amines by HPLC-MS/ MS. Public Health Laboratory. Government of Aragón (Spain). https://gobierno.aragon.es/estaticos/GobiernoAragon/ Departamentos/SanidadBienestarSocialFamilia/Sanidad/ Profesionales/13_SaludPublica/Cartera_Servicios_Laboratorio_ 20190507.pdf

21. Pietrangeli P, Federico R, Mondovì B, Morpurgo L. Substrate specificity of copper-containing plant amine oxidases. J Inorg Biochem. 2007;101:997-1004. https://doi.org/10.1016/j.jinorgbio.2007.03. 014.

22. Connah D, Westland S, Thomson MGA. Recovering spectral information using digital camera systems. Color Technol. 2001;117: 309-12. https://doi.org/10.1111/j.1478-4408.2001.tb00080.x.

23. Goldman J. Quantitative analysis on thin-layer chromatograms. Theory of absorption and fluorescent densitometry. J Chromatogr. A1973. 78:7-19. https://doi.org/10.1016/S0021-9673(01)99031-4.

Publisher's note Springer Nature remains neutral with regard to jurisdictional claims in published maps and institutional affiliations.

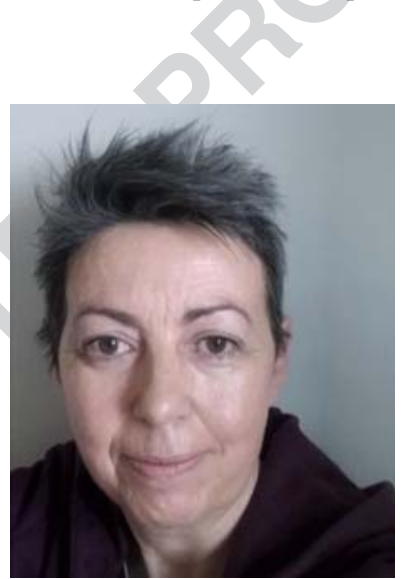

Isabel Sanz-Vicente is a member of the Nanosensors and Bioanalytical Systems Group and of the Aragon Institute of Nanoscience (INA). From 2000, she is an Assistant Professor in Analytical Chemistry at the University of Zaragoza. Her work has been focused on the use of the optic properties, intrinsic and extrinsic, of enzymes for the development of self-indicating analytical methodology, sensitive and selective, as the basis of optical biosensors.

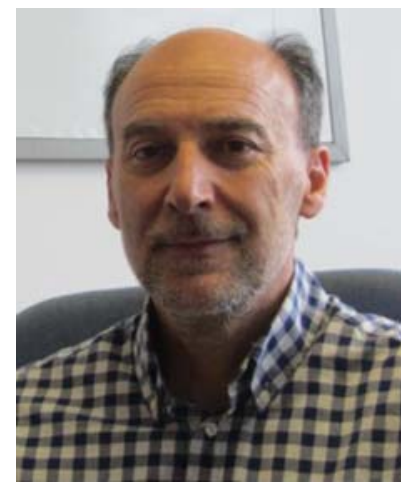

Ángel López Molinero is a Full Professor of Analytical Chemistry in the University of Zaragoza since 1987 . He was a visitor in CNRS Lyon, France, in developing ICP-AES for analytical spectroscopy. Lately, he has moved his interest to digital imagebased methods. Principles and feasibility of colorimetric detections by digital image sensors are studied by advanced chemometric methods. 
Smartphone-interrogated test supports for the enzymatic determination of putrescine and cadaverine in food

736

737

738

739

740

741

742

743

744

745

746

747

748

749

750

753

752

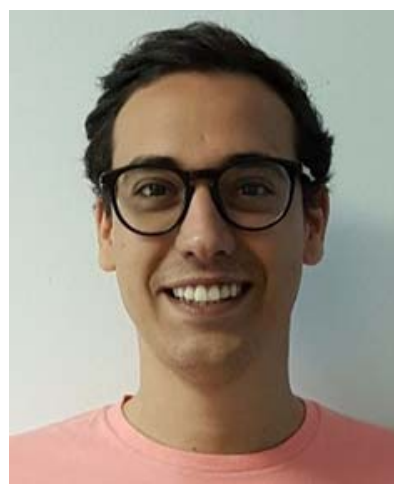

Jesús Navarro is graduated in chemistry and biochemistry from the University of Zaragoza and master in chemical science and technology with specialty in analytical chemistry. Currently, he is a $\mathrm{PhD}$ student focused on the study of optical analysis methods based on enzymatic reactions. These enzyme systems have been implemented on solid supports to perform rapid analysis methods. It is worth noting the study of chromophores and gold nanoparticles as the basis of enzyme platforms,

receiving two awards at conferences.

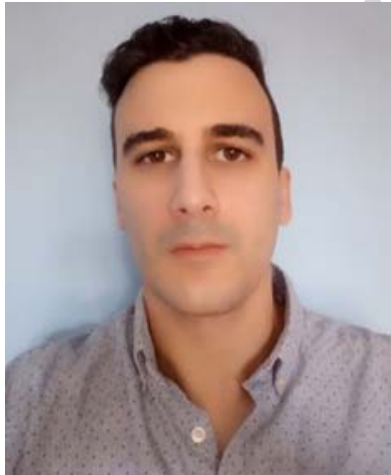

Pablo Cebrián is graduated in chemistry and master in industrial chemistry from the University of Zaragoza.

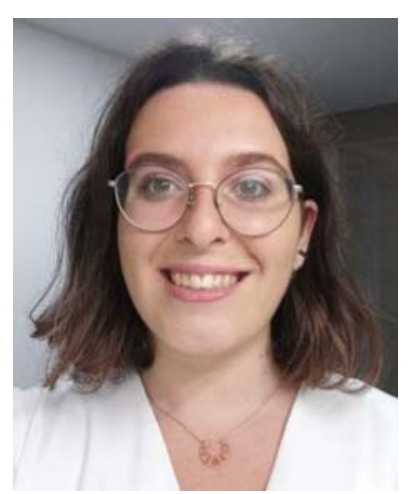

Chantal Arruego is graduated in chemistry from the University of Zaragoza.

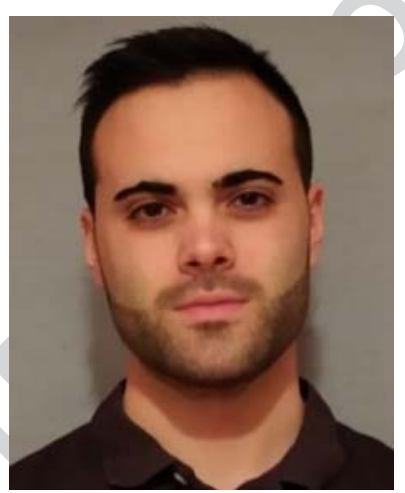

Víctor Visiedo is graduated in food science and technology from the University of Zaragoza. He is studying a European Master in Food Safety and Quality.

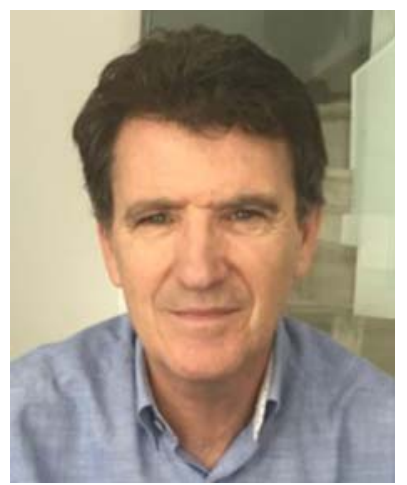

Javier Galbán is a Full Professor in Analytical Chemistry in the University of Zaragoza from 2003, where he is the coordinator of the Nanosensors and Bioanalytical Systems Group. He also belongs to the Aragon Institute of Nanoscience. His interest fields are optical (mainly fluorescence and molecular absorption) (nano)biosensors as a part of monitorization devices and point-of-care systems for clinical and food analysis.
802

803

804

805

806

807

808

809

810

811

812

813

814

815 


\section{AUTHOR'S PROOF!}

\section{AUTHOR QUERIES}

\section{AUTHOR PLEASE ANSWER ALL QUERIES.}

Q1. Please check if the section headings are assigned to appropriate levels.

Q2. Please check the modified expanded form "horseradish peroxidase" of the abbreviation "HRP" if correct.

Q3. The phrase "minor than 350" was changed to "less than 350." Please check if correct.

Q4. The presentation of section citations was modified in compliance with the journal style. Please check if all section citations are correctly presented.

Q5. Please check the modified section citation "Put and Cad determination in a tuna sample" if correct in the sentence starting "Put and Cad present in the extract of the tuna sample." 